International Journal of Biomedicine | June 2021 - Volume 11, Issue Suppl_1: Abstracts from the Third Russian International Conference "Cryo-electron microscopy 2021: achievements and prospects"

POSTER ABSTRACT PRESENTATIONS

SESSION TITLE: STRUCTURE AND FUNCTIONS OF THE TRANSCRIPTION AND TRANSLATION APPARATUS OF THE CELL

DOI: 10.21103/JJBM.11.Suppl_1.P18

\title{
Abstract P-18: Phosphorylation of RNA Polymerase II C-Terminal Domain Affects Transcript Elongation Through Chromatin
}

Sohail Akhtar $^{1}$, Elena Y. Kotova ${ }^{2}$, Nadezhda S. Gerasimova ${ }^{3}$, Vasily M. Studitsky ${ }^{2,3}$

${ }^{I}$ Molecular and Structural Biology Division, Council of Scientific and Industrial Research (CSIR)-Central Drug Research Institute, Lucknow, India

${ }^{2}$ Fox Chase Cancer Center, Philadelphia, PA, USA

${ }^{3}$ Biology Faculty, Lomonosov Moscow State University, Moscow, Russia

Background: Transcription is the central point of gene regulation where the efficient maintenance of chromatin structure during the passage of RNA polymerase (Pol II) is critical for cell survival and functioning. The phosphorylation of carboxy-terminal domain (CTD) of the large subunit (Rpb1) of Pol II plays a key role in transcription through chromatin providing the binding and dissociation of factors essential for the mRNA biogenesis. Although the regulatory effect of chromatin structure on multiple stages of transcription has been well established, the role of CTD phosphorylation itself has not been systematically addressed.

Methods: The effect of differentially phosphorylated Pol II-CTD on transcript elongation through chromatin was studied using in vitro transcription system based on mononucleosomes precisely positioned on DNA. The unphosphorylated and hyperphosphorylated Pol II-CTD were obtained using yeast genetics as well as in vitro kinase or phosphatases. Transcription rate and positions of pausing were measured using authentic elongation complexes comprising Pol II having different CTD phosphorylation states. The quantitative analysis of the transcripts was conducted using denaturing PAGE.

Results: We observed a significant difference in the transcription through chromatin depending on CTD phosphorylation level. Thus, experiments on transcription of nucleosomes with Pol II isoforms have shown that the hyperphosphorylated form more efficiently transcribes the nucleosome and leads 
to a faster accumulation of the full-length RNA product than the nonphosphorylated isoform of Pol II. The non-phosphorylated isoform of the enzyme is characterized by a stronger pause in the early nucleosomal region and a slower accumulation of the full-length RNA product.

Conclusion: Hyperphosphorylated form more efficiently transcribes the nucleosome and leads to a faster accumulation of the full-length RNA product as compared with the non-phosphorylated isoform of Pol II. A preliminary model of the effect of Pol II hyperphosphorylation on nucleosomal DNA transcription is proposed.

Key Words: RNA polymerase $\bullet$ transcription $\bullet$ nucleosome $\bullet$ C-terminal domain.

This work was supported by the Russian Scientific Foundation (project No. 1944-02013 to V.M.S.) and the Department of Science and Technology, India (Bilateral Grant Project Code No. GAP0330 to S.A.)

*Corresponding author: Sohail Akhtar.E-mail:sohail@cdri.res.in

International Journal of Biomedicine. 2021;11 Suppl 1: S19.

doi: 10.21103/IJBM.11.Suppl_1.P18

(C)2021 International Medical Research and Development Corporation 